\title{
Strokes in Young Adults
}

\author{
B. I. B. SENEVIRATNE, B. AMERATUNGA
}

British Medical fournal, 1972, 3, 791-793

\section{Summary}

Occlusion of the internal carotid or middle cerebral artery was seen in 44 young adults of both sexes from a rural population in Ceylon over a period of four years. None had hypertension, diabetes, prediabetes, or hypercholesterolaemia. There were 19 men with internal carotid occlusions, most being due to atherosclerotic thrombosis. The high incidence of atherosclerosis in these patients on a marginal diet remains an enigma, and we suggest that carbohydrate-induced hyperlipidaemia might be an important aetiological factor. There were 13 men with middle cerebral occlusions, the aetiology of which remains obscure. Occult embolism or atherothrombosis are suggested as possible causative factors. Of the 12 women five had middle cerebral artery occlusions in the last trimester of pregnancy and two had internal carotid artery occlusions in the puerperium. The pattern of ischaemic strokes in women aged 15-45 was similar to that observed in Western countries, though our patients differed ethnologically and in dietary habits.

\section{Introduction}

While working at the General Hospital, Badulla, Ceylon, we were impressed by the number of cases of non-haemorrhagic stroke in patients aged 12 to 45 years who had occlusive disease of either the middle cerebral or internal carotid vessels but no other apparent predisposing cause. We hesitated to attribute them to atherosclerosis, since this is believed to be uncommon among Orientals. It is generally rare also in Western people aged under 50 and usually found only in the presence of predisposing factors. McDowell (1967), in the Cornell-Bellevue series of 873 cases of non-haemorrhagic strokes, attributed $92 \%$ to atherosclerosis and emphasized that only $8 \%$ of the patients were aged under 50 . Analysing strokes in 56 young adults, Louis and McDowell (1967) found hypertension, diabetes, prediabetes, or raised serum lipids in nearly all.

We therefore decided to conduct a prospective study of cases of stroke in young adults caused by occlusion, confirmed by arteriography, of the internal carotid or middle cerebral arteries to find whether the occlusive lesions were due to atherosclerosis or perhaps to a local arteritis-a condition not previously recognized. We report here on 44 cases seen over the four-year period January 1967 to December 1970.

\section{Patients and Methods}

The hospital serves a predominantly rural population of mainly Sinhalese peasants engaged in agriculture and a minority of South Indian Tamils working in tea plantations, who have been settled in these parts since about 1850 . The diet of almost all the patients was marginal and the consumption of eggs, milk, and butter was negligible. They consumed hardly any coconut fat, since the palm does not grow in the hilly central district of

General Hospital, Kurunegala, Ceylon

B. I. B. SENEVIRATNE, M.D., M.R.C.P., Physician

B. AMERATUNGA, M.B., D.M.R.D., Radiologist
Ceylon and nuts from outside are too expensive for the average villager.

A total of 74 patients with stroke, aged 12 to 45 years, were admitted to the general medical wards (annual admissions 7,200 ) during the four-year period. There was no special neurosurgical unit so the cases were unselected. A stroke was defined as a sudden focal neurological deficit lasting for 24 hours or more resulting from a vascular lesion not due to direct trauma or spontaneous haemorrhage. The study was confined to strokes caused by lesions of the middle cerebral or internal carotid arteries. All the patients were seen within 72 hours of the stroke and were examined by one of us (B.I.B.S.). Care was taken to exclude any underlying disease which might have directly caused or predisposed to the stroke.

A complete haematological and cerebrospinal fluid examination, urine analysis, and electrocardiography was carried out in all cases together with measurements of fasting and postprandial blood sugar, blood urea, and serum cholesterol levels. The L.E. cell test was done in 20 cases in which collagen disease was suspected, and in five of these biopsy of muscle was also performed. Percutaneous carotid angiography was performed on the appropriate side under local anaesthesia by one of us (B.A.) within seven days in every case and within five days in most, and in 10 cases the angiography was performed on both sides. To avoid possible errors of interpretation the bifurcation of the common carotid artery was always visualized.

\section{Clinical Findings}

Arterial occlusion was found in 44 of the 74 patients admitted with a stroke; 32 were male and 12 female. Their age and sex distribution and the artery occluded are given in Tables I and II.

TABLE I-Age Group, Sex, and Artery occluded in 44 Patients with Ischaemic Stroke

\begin{tabular}{|c|c|c|c|c|c|c|c|c|}
\hline \multirow{2}{*}{\multicolumn{3}{|c|}{ Artery Occluded }} & \multicolumn{2}{|c|}{ 15-25 Years } & \multicolumn{2}{|c|}{ 26-35 Years } & \multicolumn{2}{|c|}{ 36-45 Years } \\
\hline & & & M. & F. & M. & F. & M. & F. \\
\hline $\begin{array}{l}\text { Internal carotid } \\
\text { Middle cerebral }\end{array}$ & 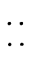 & $\because$. & $\begin{array}{l}2 \\
1\end{array}$ & $\begin{array}{l}2 \\
1\end{array}$ & $\begin{array}{l}8 \\
5\end{array}$ & 2 & $\begin{array}{l}9 \\
7\end{array}$ & $\begin{array}{l}2 \\
3\end{array}$ \\
\hline Total & .. & $\ldots$ & 3 & 3 & 13 & 4 & 16 & 5 \\
\hline
\end{tabular}
TABLE II-Mean Ages, Serum Cholesterol Levels, and Blood Pressure Ranges
among 44 Patients with Stroke due to occlusion of Internal Carotid or Middle Cerebral Artery

\begin{tabular}{|c|c|c|c|c|c|}
\hline $\begin{array}{c}\text { Artery } \\
\text { Occluded }\end{array}$ & $\begin{array}{l}\text { No. of } \\
\text { Patients }\end{array}$ & Sex & $\begin{array}{c}\text { Age } \\
\text { (Mean } \pm \text { S.D. })\end{array}$ & $\begin{array}{c}\text { Serum } \\
\text { Cholesterol } \\
(\text { Mean }+ \text { S.D. } \\
(\mathrm{mg} / 100 \mathrm{ml})\end{array}$ & $\begin{array}{c}\text { Blood } \\
\text { Pressure } \\
\text { (mm Hg) }\end{array}$ \\
\hline $\begin{array}{l}\text { Internal carotid } \\
\text { Internal carotid } \\
\text { Middle cerebral } \\
\text { Middle cerebral }\end{array}$ & $\begin{array}{r}19 \\
6 \\
13 \\
6\end{array}$ & $\begin{array}{l}\text { M. } \\
\text { F. } \\
\text { M. } \\
\text { F. }\end{array}$ & $\begin{array}{l}33 \cdot 6 \pm 7 \cdot 0 \\
30 \cdot 6 \pm 8 \cdot 9 \\
35 \cdot 6 \pm 6.8 \\
33 \cdot 0 \pm 6 \cdot 0\end{array}$ & $\begin{array}{l}194.7 \pm 15.5 \\
183.1 \pm 26.0 \\
186.1 \pm 19.4 \\
169.1 \pm 17.9\end{array}$ & $\begin{array}{l}100 / 70-140 / 85 \\
100 / 70-120 / 80 \\
100 / 70-130 / 90 \\
100 / 70-120 / 80\end{array}$ \\
\hline
\end{tabular}

In all but one of the 19 cases of internal carotid occlusion in males the artery was occluded at its origin. In 11 cases the left side was affected and in eight the right side. Seven of the 12 cases of occlusion in females were associated with pregnancy or the puerperium. Two developed sudden paralysis within a week of normal childbirth after uncomplicated pregnancy. Five had their strokes in the last trimester of pregnancy, and in each case the middle cerebral was the artery occluded.

In none of the cases of either sex was the serum cholesterol 
level above $225 \mathrm{mg} / 100 \mathrm{ml}$. The mean cholesterol levels and blood pressure ranges among the 44 patients are given in Table II.

\section{TYPICAL CASES}

Case 1.-A man aged 21 was admitted to hospital with right hemiplegia. Angiography showed occlusion of the left internal carotid artery at its origin. He recovered well and was ambulant and independent in four weeks. Repeat angiography after six months showed no change, and a short segment of the internal carotid at its origin was excised. The lumen was completely occluded and there was much intimal fibrosis and degeneration of the media-the end result of atherosclerosis.

Case 6.-A man aged 28 was admitted with dense right-sided hemiplegia and in deep coma. Angiography on admission showed occlusion of the left internal carotid. He remained in coma and died after 48 hours. At necropsy the left internal carotid artery was completely obstructed by a large thrombus on an atheromatous plaque. The left middle cerebral artery was occluded by an embolus, probably originating from the internal carotid. The resulting infarction of the left cerebral hemisphere consisted of a large haemorrhagic area of softening. The right internal carotid had early atheromatus changes at its origin. The cause of the occlusion in this case was definitely atheroma. The findings also supported the view that occlusions of the middle cerebral artery are often due to embolism from diseased extracranial vessels.

Case 18.-A man aged 45 was admitted with right hemiparesis which had been preceded by left-sided transient monocular blindness and headache. Systolic bruits were heard in the neck over the left carotid and in the epigastrium. He also gave a six-month history of progressive intermittent claudication of both lower limbs. He smoked 30 cigarettes a day. Both femoral pulses were weak and the popliteal, posterior tibial, and dorsalis pedis pulses on each side were impalpable. The blood pressure in the upper limb was $140 / 85 \mathrm{~mm} \mathrm{Hg}$. Blood cholesterol was $175 \mathrm{mg} / 100 \mathrm{ml}$. Angiography showed partial occlusion of the left internal carotid artery at its origin and an occlusion of the aorta below the origin of the renal arteries. At endarterectomy extensive atheroma, on which a recent thrombus was superimposed, was found in the left internal carotid. He made a good recovery. Surgery on the lumbar aorta was postponed. This was a case of extensive atherosclerosis in a young man whose blood cholesterol was within the normal range.

Case 19.-A man aged 45 was admitted with transient attacks of weakness of the left upper limb and aphasia. Each attack lasted two to three hours and was followed by complete recovery. $\mathrm{He}$ had had at least 10 such attacks before admission. Blood sugar and blood cholesterol were normal. Angiography showed a partial occlusion of the right internal carotid artery. The attacks stopped soon after anticoagulant therapy was instituted and he remained free of attacks for three months, after which he failed to attend the clinic. He was readmitted one year later with acute myocardial infarction. He also had right-sided hemiparesis which he said had developed three months previously. This was a case of widespread atherosclerotic disease with no hypercholesterolaemia or diabetes.

Case 43.-A woman aged 40 was admitted with sudden rightsided hemiplegia with no premonitory symptoms. Her blood pressure was $120 / 80 \mathrm{~mm} \mathrm{Hg}$. Electrocardiography and teleradiography showed no abnormality. Carotid angiography showed complete occlusion of the left middle cerebral artery. She made an uneventful recovery and in three months was back at work. Two years later she was readmitted in severe congestive cardiac failure and died within two hours. An E.C.G. on admission showed sinus rhythm and complete bundle-branch block. The necropsy findings were those of a congestive cardiomyopathy. The heart was grossly enlarged; the coronary vessels and the valves were normal. The systemic vessels were patent and free of atheroma. This case was not excluded from our series because it illustrates how an early cardiomyopathy may be the source of "occult" embolism.

\section{Discussion}

Possibly the pathogenesis of middle cerebral and internal carotid artery occlusions are quite distinct (Lhermitte et al.,
1970), though they may be related. Thus we discuss the two lesions separately.

\section{INTERNAL CAROTID OCCLUSION}

Most occlusions of the internal carotid artery at its origin are due to thrombosis on an existing atheromatous plaque (Fisher, 1951, 1954). Castiagne et al. (1970) studied the necropsy findings in 61 internal carotid occlusions in 50 patients all aged over 40 (mean aged 60 ) and concluded that $60 \%$ were due to atherosclerosis However, 13 cases $(21 \%)$ were due to embolism, the main source being the heart (mitral valve disease with fibrillation or myocardial infarction). This is not generally recognized, though occlusions of large vessels such as the femoral artery by embolism after a cardiac infarction are well known. Large emboli also occur with fungal endocarditis.

The incidence of embolism may be higher in younger patients than recorded by Castiagne et al., but we took particular care to exclude it in these patients. Sindermann et al. (1970), in a comparative study of middle cerebral and internal carotid artery occlusions, confirmed that most of those of the internal carotid at its origin were due to atherosclerosis and they found a preponderance on the left side, as we did. They suggested that carotid occlusions beyond the cavernous sinus (supraclinoid) and middle cerebral occlusions were seldom due to atherosclerosis and were the usual findings in patients aged under 40. We agree that supraclinoid occlusions are often non-atherosclerotic. Three such cases under our care (not included in this series) had tuberculous meningitis.

Trauma or infection may be important aetiological factors in children (Bickerstaff, 1964) and even in adults (Humphrey and Newton, 1960). In our series there was a history of trauma in only one case and the paralysis occurred six hours after. Angiography performed one week later on the side unaffected by the trauma showed partial occlusion of the internal carotid, and so trauma may precipitate thrombosis on an already established atheromatous plaque.

Thromboangiitis obliterans, common in Ceylon, has been foremost in our minds in considering the various forms of arteritis as possible aetiological factors. In a series of $\mathbf{4 0}$ patients with thromboangiitis under our care, in whom peripheral vascular disease was excluded, there was occlusion of the internal carotid artery in only one, and this may have been due to atherosclerosis. Though the rare possibility of thromboangiitis affecting the carotid arteries in the later stages of the disease cannot be excluded, it would be very unusual for it to begin in the carotids and present as an occlusion without other vessels being affected. On these grounds we can safely exclude thromboangiitis as an aetiological factor in our series of cases.

We may fairly assume that the basis of the internal carotid occlusion in most of these patients was atherosclerosis, despite their age. The high incidence of atherosclerosis in this type of community surviving on marginal diets remains an enigma and awaits investigation. Consumption of coconut oil (a saturated fat) was slight. Moreover, serum cholesterol levels were not significantly raised. Perhaps carbohydrate-induced hyperlipidaemia may be a more important factor, and further investigations along these lines may be worth while.

\section{MIDDLE CEREBRAL OCCLUSION}

The middle cerebral artery is a common site for embolic occlusion (Dalal et al., 1965; Fisher, 1961). Occasionally embolism may present as a gradually evolving stroke and the source of embolism may not be identified, even at necropsy. Moreover, in a recent necropsy analysis of occlusions of the middle cerebral artery Lhermitte et al. (1970) emphasized the rarity of thrombotic occlusions of the middle cerebral artery. Atherosclerotic occlusive thrombosis of the middle cerebral was 
present in only two out of 47 cases of cerebral infarct. In 20 out of 31 cases emboli had originated from extracranial vascular lesions, especially the carotids and the heart. Necropsy in one of the present cases (Case 6) showed the above, but in most angiography did not show any lesions in the carotids-though disease in the aortic arch and vessels arising from it could not be excluded.

Cardiac conditions predisposing to embolism are readily detected clinically, but an early cardiomyopathy may easily be missed (Case 43). Obscure cardiomyopathies are not uncommon in Ceylon, but the normal cardiac status in the present patients had not changed through the period of follow-up. Nevertheless, only longer follow-up could definitely exclude a cardiomyopathy.

The middle cerebral artery at its origin is a common site for atherosclerosis and the rarity of occlusive thrombosis at this site had been poorly understood. Factors leading to a thrombus formation are not yet clearly defined. Assuming that the occlusions in our cases were due to local thrombosis, perhaps special factors yet unknown in the young patients may have predisposed to a local thrombus. "Occult" embolism is an attractive alternative hypothesis for the causation of these strokes.

\section{OCCLUSIONS IN WOMEN}

Cross et al. (1968) found six cases of occlusion of the internal carotid in their Glasgow series of 31 cases of non-haemorrhagic carotid stroke associated with pregnancy and the puerperium. All except one occurred in the puerperium. In Jennett and Cross's (1967) series there were 42 non-pregnant women aged 15 to 45 of whom 11 had proved internal carotid occlusion. The general pattern of internal carotid occlusion in young women in our series was therefore similar to that seen in Western countries. Cross et al. (1968) also noted that occlusions during pregnancy were more often in the middle cerebral artery and that in the puerperium they were more often in the internal carotid.
In view of the observations of Lhermitte et al. (1970) that the middle cerebral artery is a rare site for primary thrombosis, it is tempting to postulate that fibrin clots generated elsewhere in the vascular system may lodge in the middle cerebral artery, a notorious site for embolism. This presumption is based on evidence that fibrinolysins are reduced during the second and third trimester of pregnancy (Bonner et al., 1967). Furthermore, in this situation once a fibrin embolus is impacted extension may easily occur owing to lack of fibrinolytic factors. Unfortunately the literature is not clear on the necropsy findings in this type of case, though the absence of atherosclerotic narrowing is emphasized.

Thus, as in Western countries, ischaemic strokes are not uncommon in women in Ceylon aged 15 to 45, and most of them are either pregnant or in the puerperium.

We gratefully acknowledge the help of the late Professor G. H. Cooray, of the University of Ceylon, in interpreting histological sections, and we thank Professor K. Rajasuriya, director of health services, Ceylon, for permission to report this study.

\section{References}

Bickerstaff, E. R. (1964). British Medical fournal, 2, 82

Bonner, J., McNichol, G. P., and Douglas, A. S. (1967). In Modern Trends in Obstetrics, ed. R. J. Kellar. London, Butterworths.

Castiagne, P., Lhermitte, F., Gautier, J. C., Escourelle, R., and Derouesne, C. (1970). Brain, 93, 231

Cross, J. N., Castro, P. O., and Jennett, W. B. (1968). British Medical fournal, 3, 214.

Dalal, P. M., Shah, P. M., and Aiyar, R. R. (1965). Lancet, 1, 358.

Fisher, C. M. (1951). Archives of Neurology and Psychiatry, 65, 346

Fisher, C. M. (1954). Archives of Neurology and Psychiatry, 72, 187.

Fisher, C. M. (1961). In Pathogenesis and Treatment of Cerebrovascular Disease, ed. W. S. Fields, p. 151, Springfield, Illinois, Thomas.

Humphrey, J. G., and Newton, T. H. (1960). Brain, 83, 565.

Jennett, W. B., and Cross, J. N. (1967). Lancet, 1, 1019.

Lhermitte, F., Gautier, J. C., and Derouesne, C. (1970). Neurology (Minneapolis), 20,82 .

Louis, S., and McDowell, F. (1967). Annals of Internal Medicine, 66, 932.

McDowell, F. H. (1967). In Cecil-Loeb Textbook of Medicine, ed. P. B. Beeson and $W$. McDermott, 12th edn. p. 1545, Philadelphia, Saunders. Sindermann, F., Bechinger, D., and Dichgans, J. (1970). Brain, 93, 199.

\title{
Smoking and Gastro-oesophageal Reflux
}

\author{
C. STANCIU， JOHN R. BENNETT
}

British Medical fournal, 1972, 3, 793-795

\section{Summary \\ Gastro-oesophageal sphincter pressure and intra- oesophageal $\mathrm{pH}$ has been studied in 25 chronic smokers who complained of heartburn. Smoking a cigarette invariably caused a fall in sphincter pressure, and pH measurements showed an increased tendency for reflux to occur while smoking. When lower oesophageal pH was measured overnight one-third of all reflux episodes occurred while the patients were smoking, and reflux was seen during the smoking of two-thirds of all the cigarettes consumed. It is concluded that cigarette smok- ing is a common reversible cause of gastro-oesophageal reflux.}

Gastrointestinal Unit, Royal Infirmary, Hull

C. STANCIU, M.D., Research Fellow

JOHN R. BENNETT, M.D., M.R.C.P., Consultant Physician

\section{Introduction}

Gastro-oesophageal reflux is probably the commonest form of digestive disturbances, and although it uncommonly gives rise to complications it can cause great discomfort to the patient and accounts for much clinical investigation.

The reason why reflux occurs is not entirely understood, but a competent gastro-oesophageal sphincter is the main barrier to reflux (Atkinson et al., 1957; Pope, 1967; Winans and Harris, 1967; Longhi and Jordan, 1969). The mean gastro-oesophageal sphincter pressure in patients with reflux symptoms is lower than that in people without reflux (Atkinson et al., 1957; Winans and Harris, 1967; Haddad, 1970; Benz et al., 1972) though the primary reason for weakness of the gastro-oesphageal sphincter is unknown.

Studying a recent series of patients with gastro-oesophageal reflux we found that $92 \%$ were smokers, many of whom noticed that heartburn sometimes occurred while they smoked. Moreover, we discovered that during long recordings of lower oesophageal $\mathrm{pH}$ episodes of acid reflux were frequently detected when patients smoked. Dennish and Castell (1971) found that smoking led to a fall in gastro-oesophageal sphincter pressure in normal people. We therefore studied a group of smokers who 